\title{
BEARING LOADS IN A VEHICULAR FLYWHEEL BATTERY
}

\author{
By: \\ B. T. Murphy \\ J. H. Beno \\ D. A. Bresie
}

1997 SAE International Congress and Exposition, Detroit, MI, Feb 24-27, 1997.

PR - 224

Center for Electromechanics

The University of Texas at Austin

PRC, Mail Code R7000

Austin, TX 78712

(512) $471-4496$ 


\title{
BEARING LOADS IN A VEHICULAR FLYWHEEL BATTERY
}

\author{
B.T. Murphy, D. A. Bresie, and J. H. Beno \\ The Center for Electromechanics \\ The University of Texas at Austin \\ PRC, Mail Code R7000 \\ Austin TX 78712 \\ (512) 471-4496
}

\begin{abstract}
Radial and axial rotor support bearings are critical elements in flywheel batteries for vehicle applications. This paper discusses the quantification of bearing loads required for the development of optimal bearing designs, particularly magnetic bearings. The primary contributors to bearing loads are shown to be vehicle shock, vibration, maneuvering, and gyrodynamics. Emphasis is placed on transit bus applications. Available data for each is presented, including actual measurements made on buses, and a detailed analysis of gyrodynamics.
\end{abstract}

\section{INTRODUCTION - FLYWHEEL ENERGY STORAGE SYSTEMS (FESS)}

The design of a high performance FESS for use in a vehicle poses many challenges. In order to achieve an attractive specific energy $(\mathrm{kWh} / \mathrm{kg})$, it is necessary to construct the rotor from materials with high specific strength (ultimate stress/density) [1], leading to selection of composite materials employing graphite fibers over metals. This allows a higher specific energy, and increases rotor tip speed from several hundred $\mathrm{m} / \mathrm{s}$ for high strength alloys, to over $1,000 \mathrm{~m} / \mathrm{s}$ for composites. The high tip speed, in turn, leads to an enormous increase in parasitic windage loss. To reduce windage losses to acceptable levels requires spinning the rotor in a very tight vacuum on the order of $10^{-3}$ torr [2].

The use of a tight vacuum leads to another set of design challenges of particular interest for this paper, an already difficult task for the bearings is compounded by the need for vacuum compatibility. The bearings must be capable of extremely high speed, have very low friction, be stiff to adequately constrain the rotor, have long life, and have high load capacity. These requirements frequently lead to choosing magnetic bearings for FESS applications, despite the relative immaturity of magnetic bearing technology.

This paper describes issues associated with integrating FESS on future transit buses, with particular emphasis on magnetic bearings and their loads. The transit bus in question is the Advanced Technology Transit Bus (ATTB) being developed by Northrop with funding from the Federal Transit Administration (FTA) and the city of Los Angeles. The FESS is being developed by The University of Texas at Austin Center for
Electromechanics (UT-CEM), with funding from the city of Houston, the Defense Advanced Research Projects Agency (DARPA), the State of Texas, and others. Consequently, the paper is of interest to automotive engineers as well as the FESS developmental community.

\section{SOURCES OF BEARING LOADS}

Bearing loads are generated primarily by two sources: the vehicle and the FESS itself. Since the FESS is mounted in the vehicle, it is subjected to base motion input at the mounting points. Motion of the mount points can be classified as shock, vibration, and gross vehicle motion (i.e., maneuvering). Examples of these are potholes, cobblestone pavement, and cornering, respectively. Another source of bearing loads is residual mass imbalance of high speed rotors. These four load classifications (shock, vibration, maneuvering, and imbalance) are useful since different methods of analysis and testing would be used for each. There is an important sub-classification to maneuvering, referred to as gyrodynamics. It also requires special methods of analysis. All these classifications will be discussed.

The literature contains many references of measured vibration and shock loads on vehicles caused by irregularities in the road or terrain. Most measured values are defined in terms of Power Spectral Density (PSD) plots [3]. PSD's give predicted amplitude and frequency values of road vibration that are most likely to occur under various operational situations. Data shows that road induced vibration reduces rapidly with increased frequency. Since high frequency vibration is usually easier to filter than low frequency vibration, the FESS mount points will exhibit predominately low frequencies of $0.25 \mathrm{~Hz}$ up to about $25 \mathrm{~Hz}$. Nearly all published data is for the road input at either the road surface or at the axle. No published data was found for vibration or shock on the chassis of a bus.

SHOCK - Shock events, such as potholes, are common occurrences in vehicles and are characterized by their brief transient nature. Specialized methods of analysis and test have been developed [4] [5], which focus on the impulsive aspect of the event. The effect of a shock on a system is often characterized by the peak acceleration of the system resulting from the shock. Shock inputs from the roadway are mitigated (i.e., 
filtered) by the vehicle suspension. The absolute motion of the FESS mount points resulting from shock loads are required in order to establish FESS bearing loads. Mount point motion is often represented as a transient waveform of displacement or acceleration vs. time. Ideally, the motion in three orthogonal directions would be specified for each mount point. The precise waveforms are a function of both the type and severity of the shock. In simulation, however, the same waveform is often used at each mount, and vertical, lateral and longitudinal directions are treated independently.

Shocks to the FESS can also occur due to objects bumping or striking the FESS housing or support frame, such as during collisions. Quite often, acceptance or qualification tests are performed in this manner. Northrop has specified such an acceptance test in the System Specification for the ATTB. It requires that, "The ATTB mechanical and electrical equipment shall not suffer damage or performance degradation when subjected to the shock levels of 20 gee peak, 11 ms half-sine shock pulse in each direction along three mutually perpendicular axes," [6].

VIBRATION - Vibration is distinguished from shock in that the input and response attain steady state amplitudes which are maintained for an appreciable length of time. The transients that happen when the vibration source is first turned on or off are either unimportant, or are treated using shock methods. Vibration motion of the FESS mount points can be expressed as spectral density plots of acceleration vs. frequency. Using published PSD's, engineers have reconstructed typical surface terrains for use in computer simulations that test mounting hardware performance. Not contained in this approach is the transmissibility of the bus chassis itself and its contribution to mounting loads. The tires and suspension of the bus generally attenuate the high frequency road loads.

The acceptance test specification [6], requires that "functional equipment shall operate without degradation during and after exposure to vibration as encountered in normal revenue service." The specific vibration spectrum for this functional test is shown in figure 1 , and is for a location toward the rear of the bus. From an endurance standpoint, the specification also calls for operation "without degradation after being exposed to 1,000 hours of vibration in the non-operating state." The PSD for endurance is the same as the functional requirement, except with a reduced maximum gee $/ \mathrm{Hz}$ of 0.33 .

MANEUVERING - Vehicle maneuvering refers to the net rigid body motion of the vehicle (i.e., any event that results in a change in vehicle linear momentum). Some examples are cornering, braking and accelerating. Corresponding linear acceleration of the vehicle leads directly to loads on the FESS bearings. Such accelerations are generally limited by tire adhesion to several tenths of a gee. This would be the entire extent to which vehicle maneuvers need be considered, were it not for the presence of gyroscopic action of the high speed rotor discussed in a later section.

ROTATING MASS IMBALANCE - Another source of loads for FESS bearings is residual mass imbalance of the fly-

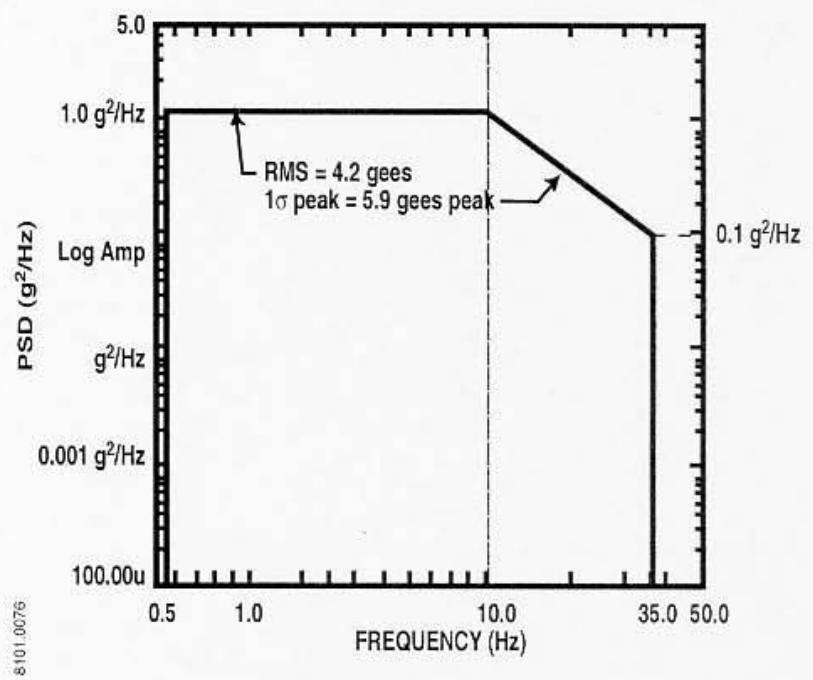

Figure 1. Specification for imposed vibration spectrum for an operating flywheel mounted toward rear of bus [6]

wheel rotor [7]. The magnitudes of these loads are proportional to the imbalance of the rotor, and are also a function of the dynamics of the rotor bearing system. Like most high speed rotors, FESS flywheels are precision balanced. After balancing, the flywheel's mass center will be within $1.3 \times 10^{-6} \mathrm{~m}$ $\left(50 \times 10^{-6}\right.$ in.) of the center of rotation. The center of rotation is dictated by the radial magnetic bearings. For this degree of balance the resulting bearing loads will be approximately $89 \mathrm{~N}$ $(20 \mathrm{lb})$, or less within the FESS operating speed range. One advantage presented by magnetic bearings is that they offer the potential to accommodate much larger mass imbalances than mentioned above. In the event that the FESS flywheel rotor does not hold its precision balance over many cycles of use, the magnetic bearing control algorithm can be adjusted to effectively rebalance the rotor [8][9].

GYRODYNAMICS - Gyroscopic effects are due directly to the relatively large angular momentum of the flywheel rotor, a vector quantity possessing both magnitude and direction. For a high speed flywheel rotor, its angular momentum vector points along the rotational spin axis and has a large magnitude. Attempts to change orientation of the flywheel spin axis (and with it the direction of the angular momentum vector), requires the application of large torques. Since nothing "touches" the flywheel except the support bearings, this torque must come from the radial bearings.

The theory of flywheel gyroscopics for road vehicles has been treated elsewhere [10]. The most important relationship describes the torque required to change the orientation of the flywheel spin axis.

$$
\text { torque }=\mathrm{P} \Omega \dot{\theta}
$$

where

$$
\begin{aligned}
& \mathrm{P}=\text { polar moment of inertia of flywheel rotor }\left(\mathrm{kg}-\mathrm{m}^{2}\right) \\
& \Omega=\text { spin speed of flywheel rotor }(\mathrm{rad} / \mathrm{s}) \\
& \dot{\theta}=\text { turning rate of the flywheel spin axis }(\mathrm{rad} / \mathrm{s})
\end{aligned}
$$


In some applications, gyroscopic torques can be large enough to affect the motion of a vehicle. Examples are ocean going ships and space vehicles [11]. In the case of a bus or car, the gyroscopic torque was shown by [10] to be too small to influence the motion of the vehicle. However, the torque is large enough to be a major contributor to loads on the radial bearings. If the flywheel is solidly anchored to the bus such that it must execute every turn experienced by the bus, the bearing loads can be more than 10 times the capacity of the radial magnetic bearings. This aspect is discussed further in a later section.

To prevent the generation of large gyroscopic torques, the orientation of flywheel rotors must be isolated from the orientation of the vehicle chassis. In practice this means that the flywheel spin axis must be vertical. This isolates the flywheel from the vehicle yaw changes (i.e., turning). In addition, some mechanism or device must support the flywheel, allowing it to pitch (nose up/down) and roll (rocking side-to-side) as freely as possible relative to the bus.

A mathematical model has been developed to study the interaction between a flywheel and a bus (fig. 2). The model represents a rigid rotating flywheel mass inside its housing, which is restrained by springs in both the pitch and roll coordinates. These springs can be attached to the vehicle frame, or they can be inertial (i.e., due to gravity). Viscous damping also acts in parallel with the springs. An addendum to this paper presents the dynamic equations of motion, using three angular coordinates, which completely describe the orientation of the flywheel. This model can be used to study a gimbal support, as well as other support designs.

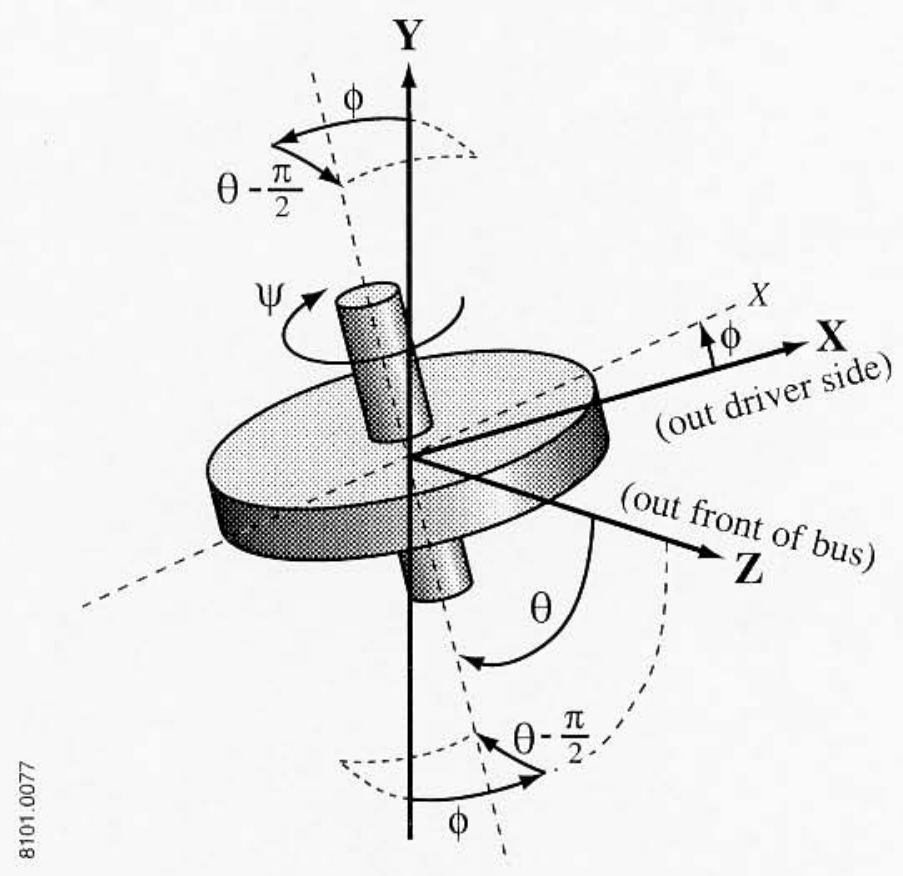

Figure 2. Mathematical model and coordinate systems used to analyze flywheel gyrodynamics
To effectively decouple the flywheel and vehicle yaw axes (which reduces gyroscopic loads on flywheel bearings during vehicle turns), the flywheel spin axis must remain nominally vertical. Thus, the support cannot be entirely neutral (i.e., if displaced from vertical and released, it must return to vertical). A restoring force is required to give the flywheel a "home" position (nominally vertical). This is easily done with springs or gravity, or both. Without a home equilibrium position, the flywheel could drift away from the vertical position, and decoupling in the yaw axis would be lost.

The analytical results obtained from the model can be summarized as follows. While at operating speed, anytime the flywheel is not in its "home" equilibrium position, it will precess about its equilibrium position in a slow circular orbit, always opposite the direction of flywheel spin. Classical analysis of a spinning top can be used to investigate the nature of this motion [12]. In practice, however, the result is simply that the flywheel will precess whenever displaced from equilibrium. The following expression for the rate of precession is derived in the addendum:

$$
\omega_{p}=\operatorname{Im}\left(\frac{(i P \Omega-C) \pm \sqrt{C^{2}-2 i C P \Omega-\left(P^{2} \Omega^{2}+4 I K\right)}}{2 I}\right)
$$

Forces or actions which can displace the flywheel from equilibrium, and thereby cause precession, include:

a) Vehicle turns in a plane (yaw), but only if the flywheel is not in its home position relative to the vehicle.

b) Vehicle pitch or roll, but only if the FESS support employs a spring and/or damper attached to the vehicle frame.

c) Vehicle lateral acceleration (turning, braking, accelerating and bumps), but only if the support employs a pendulum type spring (e.g., the flywheel center of gravity is below gimbal axis).

d) Torque on the FESS axis originating with the FESS motor-generator, but only if the stator's reaction torque vector has a component normal to the flywheel spin axis.

e) Torque on the FESS rotor applied by bearings, but only if the flywheel bearing's stator reaction torque vectors have components normal to the flywheel spin axis.

Having a home position means the flywheel will precess, an unavoidable aspect of normal flywheel operation. It is important to note that precession has several sources and can accumulate. Precession can be limited to acceptable values only through the use of damping on the pitch and roll motions. Damping must be sufficient relative to the precession inputs to minimize the probability of exceeding the allowable motion range of the support. As will be shown later, however, excessive damping can also be a major source of precession.

The focus of this paper is on bearing loads. The load on each radial bearing for a freely precessing flywheel can be expressed as follows (see addendum): 


$$
F_{b}=\frac{P \Omega \omega_{p}}{b} \theta
$$

For practical values of support parameters, this load can be small $(<45 \mathrm{~N})$. Since the load is small during free precession, efforts then focus on assuring that precession motion does not become overly large during flywheel operation.

Simulation studies performed to date have investigated loads originating with different of types of bus motion, by computing resulting flywheel precession. Different scenarios for bus motion have been devised, these generally correspond to various kinds of roads and/or driving habits. This approach is complicated somewhat by the fact that the basic precessional motion becomes linearly unstable under certain conditions of bus turning. Expressions for stability of the precessional motion are given in the addendum. In the stability analysis, the motion of the bus is not an excitation input, but rather is a moving boundary condition as seen by the flywheel. If the vehicle is viewed from above, and the flywheel is rotating counterclockwise, then right hand turns at high rates can cause flywheel precession to grow continuously. Left hand turns, on the other hand, always dissipate precessional motion.

\section{MEASURED LOADS}

Road measurements conducted at the UT-CEM by research engineers and UT Engineering Design students, have yielded estimates of design loads [13]. For these measurements, accelerometers were mounted at various places on the body of an Austin transit bus. Digital data was sampled at 24 $\mathrm{kHz}$, while the bus went over routes that included pot holes. The analyzed data from these tests showed that vibrations and shocks experienced by the bus was typically less than 1 gee. However, there is a relatively high probability that shock loads in excess of 5 gees (resulting from common obstacles such as pot holes) will occur. Shock loads as high as 8 gees were measured when the suspension "bottomed out." The two plots (fig. 3 and 4), taken from the data show typical response to road bumps.

Northrop Grumman, developer of the ATTB, has also made measurements of operational loads. Their (as yet unpublished) tests covered a broad spectrum of operational situations. Although axle acceleration of up to 13 gees occurred, high peaks were largely attenuated by the suspension and tires. Northrop concluded that peak shock loads on chassis mounted components will be less than 3 gees during transit operations. The difference between the 5 to 8 gee peaks measured in Austin and 3 gee peaks measured by Northrop is believed to be the softer suspension of the ATTB.

\section{DESIGN OPTIONS}

During the initial design stages of the FESS, UT-CEM engineers assumed that the primary peak load on the magnetic bearing would be the 3 gee shock loads imposed by road shock. The magnetic bearings are designed to transition to rolling element back-up bearings above a 3 gee level. During the design of the FESS mounting system described above, it was determined that gyroscopic effects can potentially create forces

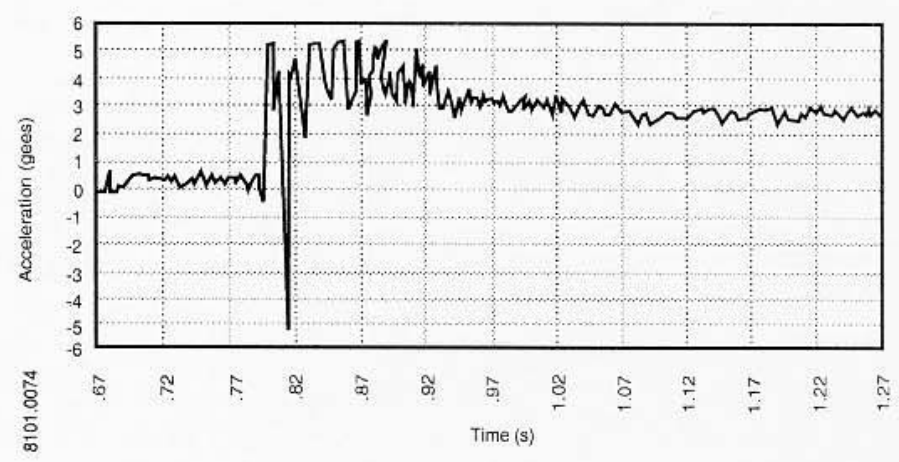

Figure 3. Chassis acceleration, typical pot-hole

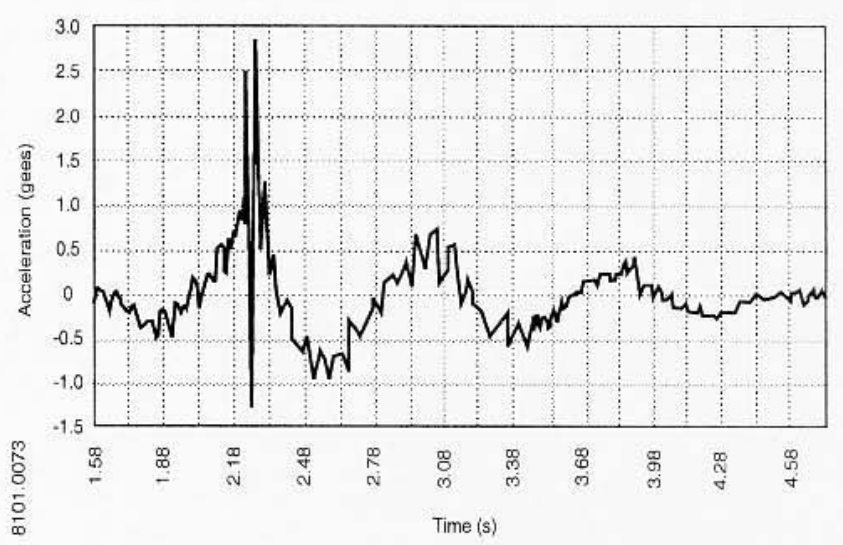

Figure 4. Chassis acceleration, typical pot-hole (expanded scale), using $33 \mathrm{~Hz}$ low pass filter

greater than those caused by a 3 gee shock. For this reason, the UT-CEM FESS mounting system is designed, not only to attenuate the shock and vibration from the road, but also to prevent gyroscopics from causing the magnetic bearings from transitioning to rolling element bearings in most situations.

Because of flywheel gyroscopics, the 3 gee capability of the magnetic bearings will be exceeded if the rotational rate of the flywheel spin axis exceeds $0.1 \mathrm{rad} / \mathrm{s}$. As a practical matter, this rate of rotation can be exceeded during vehicle operation. Exiting a steep driveway can produce a pitch rate in a transit bus of $0.24 \mathrm{rad} / \mathrm{s}$. Hitting a speed bump at high speed or going over a chuck hole can induce bus pitch of 0.23 and $0.18 \mathrm{rad} / \mathrm{s}$, (for example, see [10]). Even worse, a hard turn by the driver can cause bus roll at rates up to $1.4 \mathrm{rad} / \mathrm{s}$. All of these conditions will cause the magnetic bearings to transition to the rolling element bearings. The goal of the FESS mount design is to devise an approach that will prevent this transition.

Table 1 shows three basic design options. In the nontilting case, the flywheel cannot tilt with respect to the vehicle. Tilting concepts allow the flywheel to pitch (nose up and down) and roll (left and right) relative to the vehicle.

The non-tilting option was not a serious candidate due to the required capacity of the magnetic bearings. Of the remaining two concepts, both allow tilt. The active mount offers the 
Table 1. FESS mount design concept

\begin{tabular}{|l|l|l|}
\hline \multicolumn{1}{|c|}{ Concept } & \multicolumn{1}{|c|}{ Advantages } & \multicolumn{1}{|c|}{ Disadvantages } \\
\hline Non-tilting & $\begin{array}{l}\text { Simple, } \\
\text { compact }\end{array}$ & $\begin{array}{l}\text { Large, high } \\
\text { capacity magnetic } \\
\text { bearings }\end{array}$ \\
\hline Passive tilting & Simple & $\begin{array}{l}\text { Requires more } \\
\text { space }\end{array}$ \\
\hline Active tilting & $\begin{array}{l}\text { Low bearing } \\
\text { loads }\end{array}$ & $\begin{array}{l}\text { More space and } \\
\text { complexity }\end{array}$ \\
\hline
\end{tabular}

best overall performance. With an active mount, the flywheel housing can pitch and roll to respond to the pitch and roll motion of the bus. In this way, the actively controlled system can maintain a vertical spin axis at all times. Because the spin axis orientation remains stationary, there is no precession torque in reaction to bus tilt. An inclinometer working in conjunction with torque motors on each of two gimbal axis, for example, can keep the flywheel spin axis vertical at all times.

While the active system is the most effective way to nullify gyroscopic effects, it is more complex and expensive. The concept requires at least two torque motors along with associated electronic drivers and inertial sensors. A passive tilting mounting system concept, however, also achieves satisfactory results with simple passive hardware. It prevents bearing transitioning in all but the most extreme cases. A passive gimbal system is the basis for the UT-CEM vehicular FESS program.

EXAMPLE CONCEPTUAL DESIGN - To design a passive gimbal concept, estimates of bus roll and pitch are required. Information on roll and pitch can be found in references on roadway design [14]. Discussions with operators in Los Angeles and Houston revealed that banked roads can be as great as $10^{\circ}$. Coupled with estimated suspension roll of up to $6^{\circ}$, this give possible roll-tilting of the bus of as much as $16^{\circ}$. While bus pitch is less $\left(2.5^{\circ}\right)$, road grades are occasionally as high as $12^{\circ}$. Best estimates are that total bus tilt rarely exceeds $10^{\circ}$. Added to these relatively large tilt angles, is the possibility of displacement amplification inherent in passive suspension systems.

The center of tilt of the FESS mounting system is placed at the center of gravity of the flywheel. This prevents coupling of lateral and transverse gees into tilting torques. Fortunately this placement is also favorable to minimizing space swept by tilting of the FESS. Movements of up to $20^{\circ}$ are possible without excessive clear space required for sweep. A design value of plus or minus $20^{\circ}$ allowable tilt accommodates nearly all operational bus motion except for rollover of the bus. This $20^{\circ}$ allowable tilt also provides for motion amplification of 2 for all but the rarest conditions. During these rare conditions, transitioning to the backup bearings might occur.

The passive mount must provide for cushioning in 5 degrees of freedom: lateral, longitudinal and vertical translation, and pitch and roll rotation. To keep magnetic bearing forces low, roll and pitch motion must have a very small spring rate. The vertical, lateral, and longitudinal spring rates are selected to provide mount natural frequencies away from the dominant driving frequencies of the bus. Northrop has made estimates (table 2) of the characteristics of the ATTB. Bounce, roll, and hop are the primary excitation frequencies for the FESS mount. While the roll and pitch spring rates must be soft, excitation frequencies of the bus dictate the translational spring rates of the mount be relatively stiff. UT-CEM design engineers evaluated several approaches to the passive tilt concept using various configurations of springs and slides. The use of a gimbal to decouple rotational motion was necessary to achieve the desired combination of stiffness.

Table 2. Bus natural frequencies of the ATTB

\begin{tabular}{|l|l|}
\hline Pitch & $1.3 \mathrm{~Hz}$ \\
\hline Bounce & $1.5 \mathrm{~Hz}$ \\
\hline Roll & $3.7 \mathrm{~Hz}$ \\
\hline Bending & $4.7 \mathrm{~Hz}$ \\
\hline Twisting & $6.7 \mathrm{~Hz}$ \\
\hline Hop (Rear) & $8.0 \mathrm{~Hz}$ \\
\hline
\end{tabular}

Figure 5 shows a typical mounting system layout. This concept uses a gimbal to provide freedom to tilt in the pitch and roll directions. The use of the gimbal requires more space than other approaches, but by careful orientation of components, the concept fits within a $30 \times 30 \times 30 \mathrm{in}$. space.

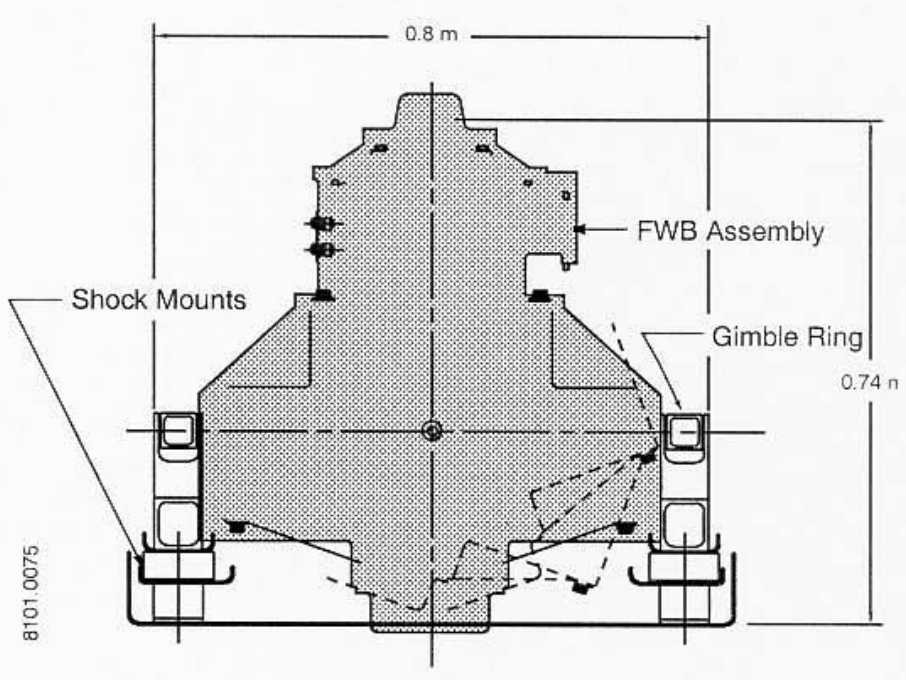

Figure 5. FESS gimbal mounting concept

\section{SUMMARY}

This paper attempts to identify the primary sources of bearing loads for energy storage flywheels targeted for use in roadway vehicles, with emphasis on transit buses. For reasons of both cost and energy efficiency, flywheel bearings are best designed to be as small as possible, and to not have more load 
capacity than necessary for the intended application. The most significant sources of bearing loads were found to be due to road shock, and flywheel gyrodynamics. In the case of flywheels supported by magnetic bearings, it is imperative to accurately quantify the anticipated bearing loads, so as to minimize the frequency of occurrence of touchdowns on mechanical backup bearings. It was shown that to avoid potentially very large loads due to gyrodynamics, a flywheel's spin axis must be oriented vertically and the flywheel must be suspended in a two-axis gimbal, so as to decouple its pitch and roll motion from that of the vehicle. Formulas for flywheel precessional motion due to gyrodynamics, and the corresponding loads on the bearings were presented. It was shown that restraining springs and dampers are necessary on the gimbal axes to keep the flywheel vertical, and to limit precession response to acceptable values. It was also pointed out that gimbal stiffness and damping parameters must be carefully chosen to avoid dynamic instability problems under certain vehicle curving conditions.

\section{ACKNOWLEDGMENTS}

Work on this project was sponsored by DARPA, Houston Metropolitan Transit Authority (MDI), and the State of Texas.

\section{REFERENCES}

[1] Stull, S. and Post, R.F., "A New Look at an Old Idea," Science and Technology Review, pp. 12-19, April 1996.

[2] Baer, M.R., "Aerodynamic Heating of High-Speed Flywheels in Low-Density Environments," proceedings of the 1978 Mechanical and Magnetic Energy Storage Contractors' Review Meeting, U.S. Department of Energy, October, 1978.

[3] B. D. Van Deusen, "Analytical Techniques for Design Riding Quality into Automotive Vehicles," SAE Transactions, vol. 76 paper 670021,1968

[4] Harris, C. M., and Crede, C. E., Shock and Vibration Handbook, 2nd Edition, McGraw-Hill, New York, 1976.

[5] Himelblau, H., Piersol, A. G., Wise, J. H., and Max, R. G., Guidelines for Dynamic Data Acquisition and Analysis, Military Handbook MIL-HDBK-XXX, 1989.

[6] Northrop Grumman, "System Specification for ATTB System, Advanced Technology Transit Bus, Final Report, Volume II," October 27, 1995.

[7] Vance, J. M., Rotordynamics of Turbomachinery, John-Wiley \& Sons, New York, 1988.

[8] Chen, H.M. and Ku, C.P.R., "Virtual Balancing of Rotor Supported by Magnetic Bearings", proceedings of the 13th Biennial ASME Conference on Mechanical Vibration and Noise; Rotating Machinery and Vehicle Dynamics, Miami, Florida, Setp. 22-25, ASME DE-Vol. 35, pp. 65-68, 1991.

[9] Beale, S. Shafai, B., LaRoccs, P., and Cusson, E., "Adaptive Forced Balancing for Magnetic Bearing Systems," proceedings of the 3rd International Symposium on Magnetic Bearings, Technomic Publishing Company, New York, pp. 601-611, 1992.

[10] McDonald, A.T., "Simplified Gyrodynamics of Road Vehicles with High-Energy Flywheels," 1980 Flywheel Technology Symposium, U.S. Department of Energy, pp. 240-258, October, 1980.

[11] Den Hartog, J.P., Mechanical Vibrations, 4th Edition, McGrawHill, New York, 1956.

[12] Marion, J. B., Classical Dynamics of Particles and Systems, 2nd
Edition, Academic Press, New York, 1970.

[13] Braychli, et. al., "Design of an Experiment to Measure Vibrations on a Public Transit Bus for Use in the Design of Vibration Isolators for a Flywheel Mounting System," Mechanical Engineering Design Project, The University of Texas at Austin, Austin, TX, 1995.

[14] Meyer, C.F., and Gibson, D.W., Route Surveying and Design, 5th Edition, Harper \& Row, New York, 1980.

[15] Ehrich, F.F., and Childs, D.W., "Self-Excited Vibration in HighPerformance Turbomachinery," Mechanical Engineering, pp. 66-79, May, 1984.

\section{NOMENCLATURE}

$\dot{\alpha}$ turning rate of the bus

$\alpha \quad$ turning angle of the bus

b radial magnetic bearing span

C gimbal viscous damping constant

$\phi \quad$ flywheel rotation about the $\mathrm{Z}$ axis (roll)

$\phi_{\mathrm{b}}$ bus roll coordinate

$\mathrm{h}$ vertical distance from gimbal axis to flywheel center of gravity

I flywheel rotor and housing transverse inertia

i $\sqrt{-1}$

$\mathrm{K}$ gimbal axis spring rate

$\mathrm{P}$ flywheel rotor polar inertia

$\theta$ flywheel rotation about the $\mathrm{x}$ axis (pitch)

$\theta_{\mathrm{b}}$ bus pitch coordinate

$\mathrm{s}$ complex eigenvalue, the imaginary part is the precession rate

t time

$\tau_{\mathrm{p}}$ precession time constant, - $1 / \operatorname{Real}(\mathrm{s})$

$\Omega$ flywheel rotational speed

W weight of flywheel rotor and housing

$\omega_{\mathrm{p}}$ Precession rate, imaginary part of s

$\mathrm{X}$ inertial axis pointing out driver side of vehicle (at $\mathrm{t}=0$ )

$\mathrm{x}$ intermediate axis, the $\mathrm{X}$ axis rotated $\mathrm{f}$ radians about the $\mathrm{Z}$ axis

$\psi \quad$ flywheel rotation about its primary spin axis

$\mathrm{Y}$ inertial axis pointing out top of vehicle (at $\mathrm{t}=0$ )

$\mathrm{Z}$ inertial axis pointing out front of bus (at $\mathrm{t}=0$ )

\section{ADDENDUM}

Figure 2 shows a schematic of the mathematical model. The three Euler angles used to specify the absolute orientation of the flywheel are shown in the figure [12]. The flywheel is initially on the $\mathrm{Z}$ axis, pointing out the front of the bus. A small angle $\phi$ rotates the flywheel about the $\mathrm{Z}$ axis. Then the angle $\theta$ is subtended an amount close to $90^{\circ}$ about the displaced $\mathrm{X}$ axis. The $\theta$ rotation causes the flywheel's primary spin axis to point downward. Finally, the $\psi$ rotation is the primary spin coordinate of the flywheel (i.e., $\dot{\psi}$ is the spin speed). Dynamic 
equations of motion are obtained directly from the Lagrangian function, as outlined in [12]. The resulting equations are given below, and are seen to be nonlinear second order differential equations. Solution of these equations was conducted numerically by direct integration using a $4^{\text {th }}$ order Runga-Kutta algorithm for various sets of system parameters and initial conditions.

The dynamic equation of motion in the $\phi$ coordinate is:

$$
\begin{aligned}
& 2(\mathrm{I}-\mathrm{P}) \dot{\theta} \dot{\phi} \sin \theta \cos \theta+\left(\mathrm{I} \sin ^{2} \theta+\mathrm{P} \cos ^{2} \theta\right) \ddot{\phi} \\
& +\mathrm{P} \ddot{\psi} \cos \theta-\mathrm{P} \dot{\theta} \dot{\psi} \sin \theta=- \text { Wh } \sin \phi \sin \theta-\mathrm{K} \phi-\mathrm{C} \dot{\phi}
\end{aligned}
$$

The dynamic equation of motion in the $\theta$ coordinate is:

$$
\begin{aligned}
\ddot{I} \ddot{\theta}= & I \dot{\phi}^{2} \sin \theta \cos \theta-\mathrm{P}(\dot{\phi} \cos \theta+\dot{\psi}) \dot{\phi} \sin \theta \\
& + \text { Wh } \cos \phi \cos \theta-\mathrm{K} \theta-\mathrm{C} \dot{\theta}
\end{aligned}
$$

The dynamic equation of motion in the $\psi$ coordinate is:

$$
\mathrm{P} \ddot{\phi} \cos \theta-\mathrm{P} \dot{\phi} \dot{\theta} \sin \theta+\mathrm{P} \ddot{\psi}=0
$$

The stiffness and damping terms in these expressions act on the absolute deflection and velocity, but really need to be relative to the bus chassis. That is, the spring constant should multiply $\left(\phi-\phi_{b}\right)$ and $\left(\theta-\theta_{b}\right)$, the deflection relative to the bus chassis, and similarly for velocities in the damping terms. With this change, these equations can be used to simulate either free precession of the flywheel, or precession response to any pitch and/or roll motion of the bus. In order to study the affect of bus turning motion on flywheel response, the input time functions for $\phi_{\mathrm{b}}$ and $\theta_{\mathrm{b}}$ are modified accordingly (see below).

Expressions (A-1), (A-2) and (A-3) can be linearized by assuming that the angles $\phi$ and $\theta-\pi / 2$ are small. Angles up to $10^{\circ}$ incur an error of only $0.5 \%$ in sin and cosine trig functions. With this assumption, (A-1) and (A-2) simplify to:

$$
\begin{aligned}
& I \ddot{\phi}+\mathrm{P} \Omega \dot{\theta}+\mathrm{C} \dot{\phi}+\mathrm{K} \phi=\mathrm{K} \phi_{\mathrm{b}}+\mathrm{C} \dot{\phi}_{\mathrm{b}} \\
& \mathrm{I} \ddot{\theta}-\mathrm{P} \Omega \dot{\phi}+\mathrm{C} \dot{\theta}+\mathrm{K} \theta=\mathrm{K} \theta_{\mathrm{b}}+\mathrm{C} \dot{\theta}_{\mathrm{b}}
\end{aligned}
$$

The coordinates $\phi$ and $\theta$ are absolute inertial coordinates for the flywheel in roll and pitch, respectively. The coordinates $\phi_{\mathrm{b}}$ and $\theta_{\mathrm{b}}$ represent roll and pitch motion of the bus. In order to incorporate turning motion of the bus into (A-1) and (A-2), expressions have been developed which transform the roll and pitch angles, $\phi_{b}$ and $\theta_{b}$, for a turned bus, back to the inertial reference frame XYZ. The flywheel absolute position and velocity are first resolved onto a coordinate frame which is fixed to the bus. Then expressions for the gimbal stiffness and damping torques are written, in the bus reference frame. These torque components are then resolved back onto the inertial frame, and inserted into (A-4) and (A-5). If $\alpha$ is the turning angle of the bus with respect to the internal frame, (A-4) and
(A-5) then become:

$$
\begin{aligned}
& \ddot{\mathrm{I}}+\mathrm{P} \Omega \dot{\theta}+\mathrm{C} \dot{\phi}+\mathrm{K} \phi+\mathrm{C} \dot{\alpha} \theta= \\
& \mathrm{K}\left(\phi_{\mathrm{b}} \cos \alpha-\theta_{\mathrm{b}} \sin \alpha\right)+\mathrm{C}\left(\dot{\phi}_{\mathrm{b}} \cos \alpha-\dot{\theta}_{\mathrm{b}} \sin \alpha\right)
\end{aligned}
$$

$$
\begin{aligned}
\mathrm{I} \ddot{\theta}- & \mathrm{P} \Omega \dot{\phi}+\mathrm{C} \dot{\theta}+\mathrm{K} \theta-\mathrm{C} \dot{\alpha} \phi= \\
& \mathrm{K}\left(\phi_{\mathrm{b}} \sin \alpha+\theta_{\mathrm{b}} \cos \alpha\right)+\mathrm{C}\left(\dot{\phi}_{\mathrm{b}} \sin \alpha+\dot{\theta}_{\mathrm{b}} \cos \alpha\right)
\end{aligned}
$$

These expressions can be readily used to compute the amplitude of steady state forced precessional response to harmonic roll or pitch motion of the bus. For example, if the bus were continuously traversing a series of hills, this would be a periodic input in the pitch coordinate. The resulting peak amplitudes of precession in both the roll and pitch coordinates can be calculated directly from (A-6) and (A-7).

In (A-6) and (A-7), the stiffness and damping constants for the two gimbal axes were taken to be equal (i.e., $\mathrm{K}_{\mathrm{O}}=\mathrm{K}_{\theta}=\mathrm{K}$ ), but they do not need to be. If the gimbal parameters are symmetric, these two expressions can be combined into one via a substitution.

$$
\begin{aligned}
& \text { Letting } z=\phi+i \theta \\
& I \ddot{z}-i P \Omega \dot{z}+C \dot{z}+K z-i C \dot{\alpha} z= \\
& K\left(\phi_{b} e^{i \alpha}+\theta_{b} e^{i \alpha}\right)+C\left(\dot{\phi}_{b} e^{i \alpha}+\dot{\theta}_{b} e^{i \alpha}\right)
\end{aligned}
$$

The homogeneous form of (A-8), yields an eigensolution for the complex eigenvalues of the rotating flywheel. The imaginary part of the eigenvalue is the damped precession frequency. The reciprocal of the imaginary part, times $2 \pi$, is the orbital period in seconds. The reciprocal of the real part is the exponential time constant of free decay for the precession motion. The real part should be negative to indicate that precession decays. Should the real part be positive, the precession amplitude would grow without bound.

$$
s=\frac{(i P \Omega-C) \pm \sqrt{C^{2}-2 i C P \Omega-\left(P^{2} \Omega^{2}+4 I K\right)+4 i l C \dot{\alpha}}}{2 I}
$$

The precession rate and time constant are defined as:

$$
\begin{gathered}
\omega_{\mathrm{p}}=\mathrm{I}_{\mathrm{m}}(\mathrm{s}) \quad(\mathrm{rad} / \mathrm{s}) \\
\tau_{\mathrm{p}}=\frac{-1}{\operatorname{Re}(\mathrm{s})}
\end{gathered}
$$

The plus sign in (A-9), yields an eigenvalue of very low frequency, having an orbital period on the order 1 to 2 minutes per cycle for a flywheel sized for a bus. The minus sign results in a high frequency eigenvalue, on the order of 100 orbits per second. It is the slow eigenvalue which is observed as the flywheel responds to motion of the bus. 
The last term on the left hand side of (A-8) represents a coupling mechanism between turning of the bus and flywheel precession. This coupling acts by way of gimbal damping. It can be shown that this term can cause the real part of the slow eigenvalue to become positive, and the flywheel to become dynamically unstable. This happens when $\Omega$ and are opposite in sign, and the turning rate $\&$ is above a certain threshold value. The threshold value depends on the gimbal parameters, and the turning rate. The threshold value can fall within the range of practical bus turning rates. So care must be taken when selecting gimbal parameters. In the field of high performance turbomachinery, dynamic instability mechanisms similar to this exist in many forms [15].

Through (A-6) and (A-7), an expression can be developed for the flywheel bearing loads during any precession motion. The flywheel precession is a simple circular orbital motion, slowly spiraling inward when stable, or outward when unstable. Again, the precession is slow with the orbital period on the order of minutes per orbit. By substituting the conditions for circular precession into (A-6) and (A-7), the following expression for torque is obtained (the bus is stationary).

$$
\text { Free Precession Torque }=\mathrm{P} \Omega \omega_{\mathrm{p}} \theta
$$

The torque depends on the gimbal parameters by way of the precession rate $\omega_{\mathrm{p}}$. It is also proportional to $\theta$, the amplitude of precession. To convert this to load on the flywheel radial bearings, this torque is divided by the bearing span.

$$
\text { Free Precession Bearing Load }=\frac{P \Omega \omega_{p} \theta}{b}
$$

When the bus pitches or rolls suddenly (e.g., railroad tracks and curbside storm drains), a relatively large velocity of pitch or roll will occur. Since the flywheel will essentially maintain a constant orientation during such a short transient event, the gimbal damping constant, $\mathrm{C}$, times the event velocity $\dot{\theta}$ will produce a torque, and associated bearing loads.

$$
\text { Fast Excursion Bearing Load }=\frac{\mathrm{C} \dot{\theta}}{\mathrm{b}}
$$

If this type of load proves to be overly large, then some type of load limiting feature must be incorporated into the damper device. For example, standard automotive shock absorbers have internal relief valves to limit damping forces. 